\title{
Transcatheter aortic valve implantation for patients with lung cancer and aortic valve stenosis
}

\author{
Takashi Sakai ${ }^{1,2}$, Kazuyuki Yahagi ${ }^{3}$, Sumio Miura ${ }^{3}$, Tatsuhiro Hoshino ${ }^{1}$, Toshiya Yokota ${ }^{1}$, Kengo Tanabe ${ }^{3}$, \\ Shingo Ikeda ${ }^{1}$ \\ ${ }^{1}$ Department of Thoracic Surgery, Mitsui Memorial Hospital, Tokyo, Japan; ${ }^{2}$ Department of Thoracic Surgery, Toho University Graduate School of \\ Medicine, Tokyo, Japan; ${ }^{3}$ Department of Cardiology, Mitsui Memorial Hospital, Tokyo, Japan \\ Correspondence to: Kazuyuki Yahagi, MD. Department of Cardiology, Mitsui Memorial Hospital Kanda-Izumicho 1, Chiyoda-ku, Tokyo 101-8643, \\ Japan. Email: yyahakazu@gmail.com.
}

\begin{abstract}
Many patients who require lung resection have cardiovascular and cerebrovascular comorbidities. It has been recommended that surgical aortic valve replacement (SAVR) should precede lung resection in patients with severe aortic valve stenosis (AS). However, by first undergoing transcatheter aortic valve implantation (TAVI), the patient may undergo lung resection more safely. We present two patients with both severe AS and lung cancer who underwent TAVI and lung resection without any complications.
\end{abstract}

Keywords: Transcatheter aortic valve implantation (TAVI); aortic valve replacement (AVR); aortic valve stenosis (AS); lung cancer

Submitted Mar 17, 2018. Accepted for publication Apr 06, 2018.

doi: $10.21037 /$ jtd.2018.04.83

View this article at: http://dx.doi.org/10.21037/jtd.2018.04.83

\section{Introduction}

In patients with resectable lung cancer and severe aortic valve stenosis (AS), it has been recommended that treatment of AS should precede lung resection (1). If surgical treatment of both lung cancer and AS had increased risk, some cases underwent careful preceding lung resection. However, major adverse cardiovascular events (MACEs) occur in more than $5 \%$ of high-risk non-cardiac surgery cases, including thoracic surgery (1-3). By undergoing preceding transcatheter aortic valve implantation (TAVI), some patients with resectable lung cancer and severe AS could then safely undergo lung resection. We present two cases of high-risk patients who were treated for both severe AS and lung cancer by performing TAVI and lung resection.

\section{Case presentation}

\section{Case 1}

An 80-year-old woman was referred to our hospital with symptomatic AS, severe heart failure, and a left lower lung tumor noted on chest X-ray and computed tomography (CT) (Figure 1). The tumor was suspicious for lung cancer, but endobronchial or CT-guided biopsy could not be performed because of severe heart failure. She was also diagnosed with severe AS using transthoracic echocardiography, with an aortic valve area (AVA) of $0.6 \mathrm{~cm}^{2}$, peak velocity of $5.9 \mathrm{~m} / \mathrm{s}$, and mean trans-aortic pressure gradient (PG) of $80 \mathrm{mmHg}$.

Treatment of AS was considered before lung resection, but surgical aortic valve replacement (SAVR) was considered inappropriate because of severe frailty and a prospective perioperative mortality rate of $23.7 \%$, calculated using the Society of Thoracic Surgery risk score (STS score). Furthermore, she had other comorbidities including marrow dysplasia syndrome, hypertension, and autoimmune hepatitis during steroid therapy. Therefore, our heart team, including a cardiologist, cardiovascular surgeon, and anesthesiologist considered her a good candidate for TAVI. TAVI was performed transfemorally using an Edwards SAPIEN 3 20-mm Transcatheter Heart Valve (Edwards Lifesciences, Irvine, California, USA) without extracorporeal circulation (Figure 2), and her heart failure 

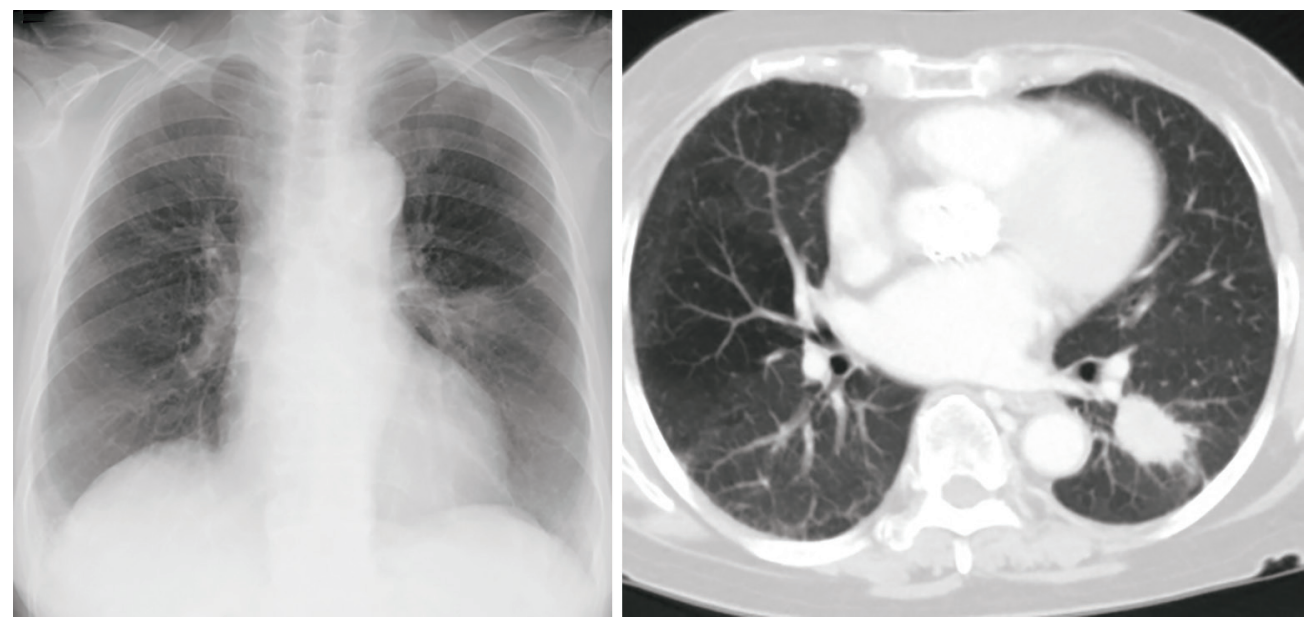

Figure 1 Chest X-ray and CT in case 1. Lung tumor is present in the left lower lobe. CT, computed tomography.

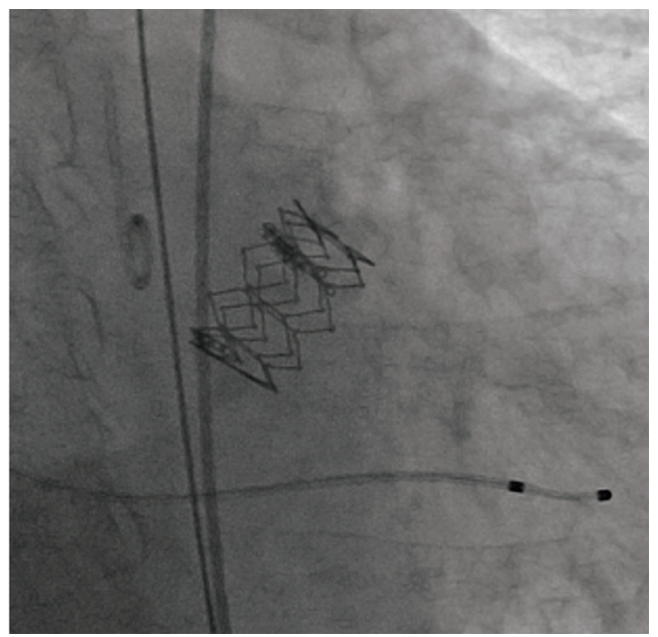

Figure 2 Intraoperative view of TAVI in case 1. TAVI, transcatheter aortic valve implantation.

improved significantly. Because she was diagnosed with primary lung adenocarcinoma, cT2aN0M0 stage IB by bronchoscopic wash cytology, video-assisted thoracoscopic Surgery (VATS) left lower lobectomy and selective lymph node dissection were performed 38 days after TAVI. She was prescribed aspirin and clopidogrel following TAVI, and a continuous heparin infusion during the perioperative period instead of dual-antiplatelet therapy, which was stopped 1 week before lung resection. She was discharged 11 days after lung resection without any complications. The pathological stage was $\mathrm{pT} 1 \mathrm{cN} 1 \mathrm{M} 0$ stage IIB, and she remains well without recurrence at 1-year follow-up.

\section{Case 2}

A 75-year-old man was referred to our hospital with a right lower lung nodule noted on chest X-ray and CT at another hospital (Figure 3). The nodule was diagnosed as primary lung adenocarcinoma, cT1bN0M0 stage IA2, using CTguided needle lung biopsy. Lung resection was planned, but asymptomatic severe AS was found on echocardiography, with an AVA of $0.8 \mathrm{~cm}^{2}$, peak velocity of $5.0 \mathrm{~m} / \mathrm{s}$, and PG of $54 \mathrm{mmHg}$. Because SAVR was high risk, with an STS score of $4.3 \%$, and because of comorbidities of paraplegia due to compression fracture and untreated renal cancer, the heart team decided to perform TAVI before lung resection. TAVI was performed transfemorally using an Edwards SAPIEN 3 23-mm Transcatheter HeartValve without extracorporeal circulation (Figure 4); VATS right segment six resection and selective lymph node dissection were performed 44 days after TAVI. Aspirin and clopidogrel were prescribed following TAVI, and heparin was continuously infused during the perioperative period instead of dual-antiplatelet therapy, which was stopped one week before lung resection. He was discharged 10 days after lung resection without any complications. The pathological stage was $\mathrm{PT} 1 \mathrm{bN} 0 \mathrm{M} 0$ stage IA2, and he remains well without recurrence at 2-year follow-up.

\section{Discussion}

TAVI has become standard treatment for AS patients judged to be at high risk of SAVR (4). Before TAVI was introduced, SAVR was recommended prior to lung resection in high-risk patients 


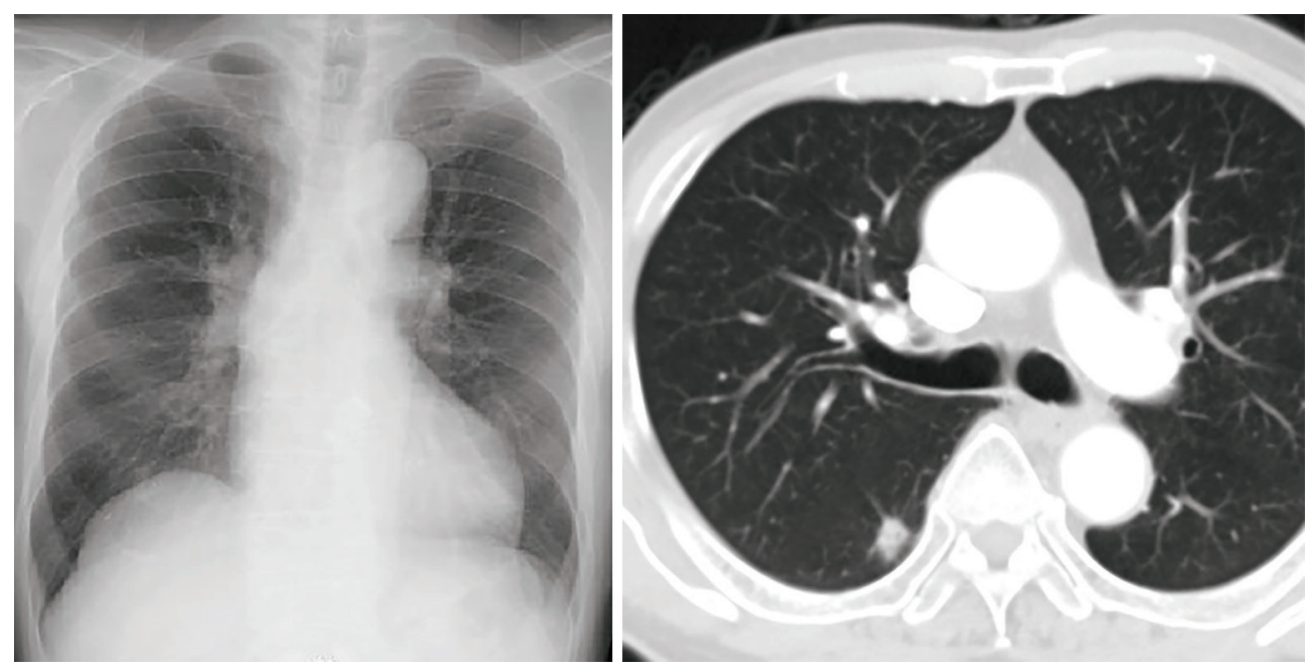

Figure 3 Chest X-ray and CT in case 2. Lung tumor is present in the right lower lobe. CT, computed tomography.

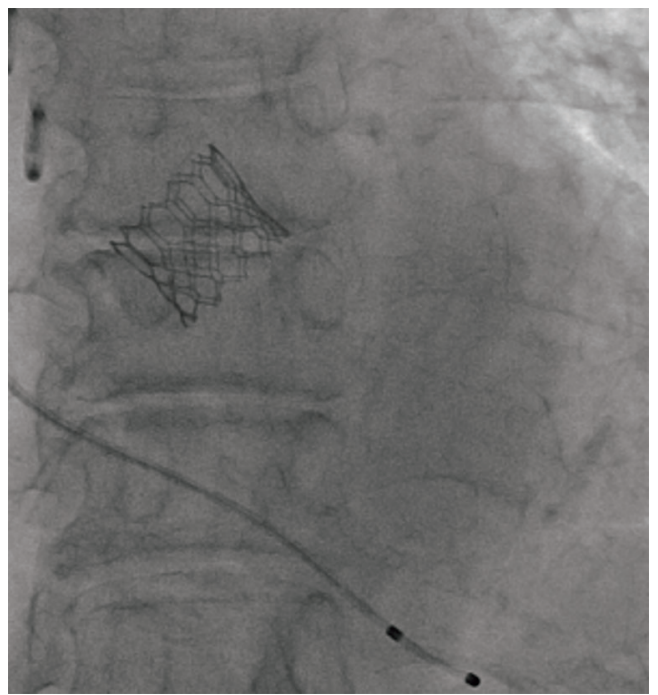

Figure 4 Intraoperative view of TAVI in case 2. TAVI, transcatheter aortic valve implantation.

with lung cancer and severe AS. However, some patients were considered high risk for surgical treatment of both lung cancer and AS. These patients had to decide between treatment of lung cancer with surgical resection before treatment of severe AS or non-surgical therapy including chemo-radiation therapy and best supportive care. Even if those patients underwent lung resection, MACE had a high probability of occurrence in the perioperative period. Thoracic surgery is classified in the high-risk MACE group, with a probability of $>5 \%(1,2)$, and some studies reported that major cardiac complications of lung resection without cerebrovascular complications occurred in $3.3 \%$ to $3.4 \%$ of cases $(3,5,6)$.

As compared to SAVR, TAVI is less invasive and does not require extracorporeal circulation. A less-invasive procedure is preferred for patients with severe AS and lung cancer, enabling early recovery following TAVI, and AVR without extracorporeal circulation may decrease lung inflammatory response and improve the cancer prognosis (7).

Antiplatelet therapy has been recommended after TAVI. Although the benefit is unclear, heparin bridging from antiplatelet therapy during the perioperative period is empirically performed in many facilities. One meta-analysis showed that continuation of antiplatelet therapy in noncardiac surgery had minimal bleeding complications, with no difference in thrombotic complications (8).

Currently, there is no evidence for the usefulness and safety of TAVI in AS patients with lung cancer or for continued antiplatelet therapy in the perioperative period for lung resection after TAVI. Therefore, more evidence is required to improve clinical outcomes in patients with lung cancer and severe AS.

\section{Acknowledgements}

None.

\section{Footnote}

Conflicts of Interest: The authors have no conflicts of interest to declare. 
Informed Consent: Written informed consent was obtained from the patient for publication of this manuscript and any accompanying images.

\section{References}

1. Fleisher LA, Beckman JA, Brown KA, et al. ACC/ AHA 2007 Guidelines on Perioperative Cardiovascular Evaluation and Care for Noncardiac Surgery: Executive Summary: A Report of the American College of Cardiology/American Heart Association Task Force on Practice Guidelines (Writing Committee to Revise the 2002 Guidelines on Perioperative Cardiovascular Evaluation for Noncardiac Surgery): Developed in Collaboration With the American Society of Echocardiography, American Society of Nuclear Cardiology, Heart Rhythm Society, Society of Cardiovascular Anesthesiologists, Society for Cardiovascular Angiography and Interventions, Society for Vascular Medicine and Biology, and Society for Vascular Surgery. Circulation 2007;116:1971-96.

2. Kristensen SD, Knuuti J, Saraste A, et al. 2014 ESC/ ESA Guidelines on non-cardiac surgery: cardiovascular assessment and management: The Joint Task Force on non-cardiac surgery: cardiovascular assessment and management of the European Society of Cardiology (ESC) and the European Society of Anaesthesiology (ESA). Eur Heart J 2014;35:2383-431.

3. Lee TH, Marcantonio ER, Mangione CM, et al. Derivation and Prospective Validation of a Simple Index for Prediction of Cardiac Risk of Major Noncardiac Surgery. Circulation 1999;100:1043-9.

4. Nishimura RA, Otto CM, Bonow RO, et al. 2017 AHA/ ACC Focused Update of the 2014 AHA/ACC Guideline for the Management of Patients With Valvular Heart Disease: A Report of the American College of Cardiology/ American Heart Association Task Force on Clinical Practice Guidelines. Circulation 2017;135:e1159-95.

5. Gopaldas RR, Bakaeen FG, Dao TK, et al. Video-assisted thoracoscopic versus open thoracotomy lobectomy in a cohort of 13,619 patients. Ann Thorac Surg 2010;89:1563-70.

6. Ferguson MK, Celauro AD, Vigneswaran WT. Validation of a modified scoring system for cardiovascular risk associated with major lung resection. Eur J Cardiothorac Surg 2012;41:598-602.

7. Suzuki S, Usui A, Yoshida K, et al. Effect of cardiopulmonary bypass on cancer prognosis. Asian Cardiovasc Thorac Ann 2010;18:536-40.

8. Columbo JA, Lambour AJ, Sundling RA, et al. A Metaanalysis of the Impact of Aspirin, Clopidogrel, and Dual Antiplatelet Therapy on Bleeding Complications in Noncardiac Surgery. Ann Surg 2018;267:1-10.

Cite this article as: Sakai T, Yahagi K, Miura S, Hoshino T, Yokota T, Tanabe K, Ikeda S. Transcatheter aortic valve implantation for patients with lung cancer and aortic valve stenosis. J Thorac Dis 2018;10(5):E387-E390. doi: 10.21037/ jtd.2018.04.83 